\title{
An Accessment of the Management of Post Operative Pain in Children Admitted to the Pediatric Surgery Unit of the Korle-bu Teaching Hospital (kbth)
}

\author{
Article by Priscilla Mawuli Awo Ekpale \\ Msc Clinical Research, Texila America University \\ Email:-priscillaekpale@gmail.com
}

\section{Background}

The oxford dictionary defines pain as a "highly unpleasant physical sensation caused by illness or injury". It is also defined by the International association of pain as "an unpleasant sensory and emotional experience associated with actual or potential tissue damage" (Fisher, 2000). Now internationally it has been accepted that, pain should be expected, carefully and efficiently restricted in children irrespective of how old or mature they are and how severe their source of pain is considered to be (Fisher \& Morton 1998).

A number of researches have been done in the area of pediatric pain and this has led to an improvement in the understanding of pain in children by both their parents or guardians and their clinical health care team. This not withstanding there is still some amount of challenge with the evaluation of pain in children.

Even though internationally so many studies have been conducted in the area of pediatric pain assessment and management, in Ghana there has been little or no study conducted in this area. Even obtaining local information about the assessment of post operative pain in children was not fruitful. As a result one cannot prove that post operative pain in children in Ghana who undergo one surgery or another receives the best control therapy.

This study is to assess the management of post operative pain in children in the biggest tertiary hospital in Ghana and information gathered could inform further studies in this area that can help improve the care given to children when it comes to the management of their post operative pain.

\section{Literature review}

There have been the believe of certain misconceptions that have influenced the under management of pain in children. For instance, neonates and infants are thought to have under developed nervous system and for that reason their ability to sense pain or feel pain is lower than that of adults but this is not true since research has proven that structures needed for perception of pain in humans are present around the 25th week of gestation and so the perception of pain can occur even intrauterine (Anand and Carr, 1989).

A lot of changes have occurred in the management of pain since it was first reported that children's pain is under treated. There have been advances like a good understanding of pain in children during their developments and how to manage acute pain in children (Howard. R.F., 2003). This acquired knowledge has not been widely utilized or translated into custom clinical practice. Recently it was discovered that the pain experienced in early part of a child's life may lead to long term consequences (Howard. R.F., 2003). It has been realized that factors like the timing of pain, degree of injury and the nature of analgesia administered may be important determinants of the long term outcome of infant pain (Howard. R.F, 2003)

Post operative pain defined "as a sudden or long persisting pain that is experienced after a surgical procedure" (www.articlesbase.com). Post operative pain is an expected phenomenon. However, its passage beyond acceptable limits is a common and costly experience (Coll et al, 2004) 
The process of post operative pain management is always initiated before the surgery is done and this is because, it has been realized that good pain prevention reduces the severity of pain felt after the surgery has been done. Even though there is fast progress in the management of postoperative pain in children, children who have under gone surgery do not have their pain after the surgery being well managed because of complexity in the evaluation of the child level of pain and also fear of the possible addictive effect of pain relievers that belong to the opiod group (Lee and Jo, 2014). It is agreed that it is a bit difficult to have a faultless pain assessment method for children and pain relieving medications that do not have any adverse effect at all on them but a good assessment of the child's pain and a good pain relieving approach designed by considering how young the child is, the kind of surgery that the child is to undergo, the kind of disease condition that the child had that necessitated the surgery with administration of a combination of the different pain relieving medications can improve the reduction of post operative pain in children (Lee and Jo, 2014)

Research has proven that considerable pain stimulation devoid of appropriate analgesia, will not only cause intolerable pain at the time of the stimulation but will result in the production of 'pain memory' which subsequently produces an inflated response to any form of stimulated pain (Taddio A et al, 1994) (Taddio A et al, 1995) (Taddio A et al, 1997)

Two decades ago, there was a survey that revealed that $40 \%$ of children that had undergone one kind or another surgical procedure felt moderate or severe postoperative pain and out of this group, $75 \%$ of them received analgesia that was not enough to control their pain (Marther L, Mackie J, 1983).

A survey conducted by Lawrence Marther and Josephine Mackie in 1983 came up with findings that indicated there was some level of prevalence of post operative pain perception in children. It had a study population of 170 children who had undergone a surgery of a sought. Out of the number who had pain after the surgery, $16 \%$ did not receive any analgesic. 39\% of patients who needed opiod analgesics did not receive it and instances where the patient needed both opiod and non - opiod analgesics, there was the use of just the non- opiods in 25\% of that group of patients (http://www.articlesbase.com). Another finding from this survey confirmed that post operative pain in children was not well managed.

In Sweden there was a study conducted by M Karling et al, 2007 to access the incidence of postoperative pain in children, the inadequate treatment of pain in children and also a look at the pain management structure in the country. It was a nationwide survey and it resulted in the following findings; $23 \%$ of children on admission at hospitals had moderate to severe post operative pain. $45 \%$ of the clinicians and nurses who were respondents attested to the fact that post operative pain management in children could be done better.

Elizabeth Cummings et al also conducted a study on the prevalence and source of pain in pediatric patients. It involved the interviewing of some parents and their children on admission. As part of findings from the above study, it was realized that children were not receiving enough analgesia therapy. Neither the clinical care givers nor the parents of the children were seen by the children as helping with analgesia care. This study also confirmed that many of the children endured unnecessary height of pain whilst on admission (Cummings et al, 1996)

The process of managing pediatric post operative pain has seen much improvement over the last ten years since there came into existence of practical methods and valid tools which aid in assessing pain in children. At this point I must admit that the level of work or effort put into the management of postoperative pain in adults is so much higher than that invested in to the control of post operative pain in children who undergo one surgery or the other and this is attributable to the fact that only little is known clinically about pain in children, there is still much research work that has to be conducted in this area and the fear of having children suffer the adverse effects of opiod used as pain reduction medication by certain pediatricians (Lee and Jo, 2014) 


\section{Pain accessment in children}

The assessment and treatment of pain in children and its functional consequence present a considerable unmet challenge. The assessment of pain in children can be very difficult and this has resulted in the design of different pain assessment tools for all the various age ranges of children (D Baroudi, 2007) Most scores allocate a numerical value to one of the following dimensions: cognitive, physiological, sensory, behavioral, and even facial expression (D Baroudi, 2007).

Among the pain assessment tools are the CR IES (Jacques, 2009), FLACC (Jacques, 2009), Mc Gill Pain Questionnaire (Jacques, 2009), Numerical Rating Pain scale (Jacques, 2009), Wong Baker faces pain scale (Jacques, 2009), Comfort Observer pain scale (Jacques, 2009),

CRIES (Krechel and Bildner, 1995)

This is a pain scale that is used to access pain in neonates and infants who are six months and below. It is an observer-rated tool which access at the subject's cries characteristics, oxygenation, vital signs facial expression and sleeplessness (Jacques, 2009)

\section{FLACC}

This is an acronym for face, legs activity, crying and consolability. This scale is an observerrated scale which is made for children within the ages of 2years -7years. It can be used even on adult patients who cannot communicate their pain verbally. The assessment is between 0-10 (Jacques, 2009). A study was done in 1996 to access the validity of accessing post operative pain in children using the FLACC and it was realized that, it provided a simple structure for quantifying pain behaviors in children who may not be able to put into words the presence or severity of pain (Markel et al, 1996)

\section{Numerical rating pain scale}

The most common pain assessment tool used in the hospital settings is this one. This tool is designed such that patient can either verbally or by placing a mark on the scale to indicate his or her level of pain from 0 to 10 . This tool is usually administered to children who are nine years and above (Jacques, 2009)

Wong baker faces pain scale (Wong et al, 2001)

This tool puts together pictorial and numerical presentations to enable the patient rate his or her pain level. It has six faces in all with each face assigned a numerical value (Jacques, 2009)

Comfort pain scale (Jacques, 2009)

This tool is used when the patient whose pain is to be accessed cannot verbally describe his or her level of pain nor can he or she rate the level of pain. It can also be applied to children, adults with cognitive impairment patients with learning disabilities and patients in ICU under sedation. The scale is rated from 9-45

\section{Mc Gill pain scale (Jacques, 2009)}

This scale utilizes the ranking of a painful event with words in groups provided. Once the patient ranks the pain, the one administering the questionnaire allocates a pain rating index which is a numerical score to the patient rating.

A precise evaluation of pain in the diverse age groups in children and the efficient control of postoperative pain are continuously being developed. 
South American Journal of Clinical Research

Special Edition 2016

\section{Medications used in pediatric post operative pain management}

Management of pain and anguish should be the main concern for all clinicians. It has been proven as wrong in initial reviews that the inadequate treatment of pain in infants and children is not right. (Walco et al, 1994). In the 1970's and 1980's, there were surveys done and these indicated that infant and children were less likely to receive postoperative analgesics than adults (Schechter et al, 1986).

In children, the pharmacokinetics and pharmacodynamics of analgesics change during their development as a result, different hepatic-enzyme systems for drug metabolism mature at different rates as they develop (* Currently the use of patient - controlled systemic administration of opiod pain relievers in combination with the administration of nonsteroidal anti-inflammatory (NSAIDS) pain relievers as well as the use of local pain relievers at the site of operation either with or without the addition of adjunctive therapy have resulted in an improved and efficient control of post operative pain in children.

\section{Opiod analgesics}

It has been realized that when opiod analgesics are dosed well and monitored well after administration, they are useful in controlling moderate to severe post operative pain in children (Matthews, 2008). The age of children influences their response to opiod analgesics when they are given because it varies very much by age (Matthews, 2008). In neonates there is evidence that opiod analgesics can cause respiratory distress due to the fact that their respiratory system is not fully developed (Goldschneider et al, 2001)

\section{Non - opiod analgesics}

These include Acetaminophen, Non-Steroidal Anti-inflammatory Drugs (NSAIDS) and they are used most often as first choice drugs for the management of mild to moderate pain in children (Sutters et al, 2004). These groups of analgesics are readily available and have relatively safer spectrum of use so are usually used in the management of pediatric pain in general.

\section{Acetaminophen}

This is a metabolite of Phenacetin. It is the most widely used antipyretic used all over the world. Acetaminophen alone is a weak analgesic agent when used in the management of post operative pain (Kokinsty and Thornberg, 2003). Recently it has been shown by researches that the dosage of acetaminophen required to provide analgesia is higher than the traditional dosages used for controlling pyrexia in children, in addition it has been proven that the bioavailability of rectally administered acetaminophen is lower than the orally administered acetaminophen. The Intravenously administered acetaminophen (propacetamol) is said to provide the best bioavailability of acetaminophen. (Kokinsty and Thornberg, 2003).

\section{NSAIDS}

Over the last 10 years, NSAIDS have been very useful in the management of post operative pain in children. Usually NSAIDS are effective in the management of mild to moderate post operative pain in children (Kokinsty and Thornberg, 2003).

In Ghana most hospitals do not have any pain management protocol for children. The use of pain assessment tools in pain assessment is not common but pediatric surgery has developed so much that most pediatric surgery cases of Ghanaian children are treated in Ghana. Since post operative pain management is very necessary this study seeks to know how post operative pain is assessed and managed in Ghana and to expose the areas that need to be looked at. 


\section{Aim}

TO ACCESS THE POST OPERATIVE PAIN MANAGEMENT NEED OF PEDIATRIC PATIENTS ADMITTED TO THE PEDIATRIC SURGERY WARD OF THE KBTH.

\section{Objectives}

1. WHAT GUIDES THE SELECTION OF ANALGESICS USED AT THE PEDIATRIC SURGERY WARD OF KBTH

2. HOW WELL IS THE POSTOPER ATIVE PAIN OF PATIENTS CONTROLLED?

\section{Method}

The Pediatric surgery unit of the Korle -Bu Teaching Hospital will be the study site. It is a unit with a bed capacity of 27 . It has a nursery capacity of 10 , a ward for toddlers and another ward for young children as well as older children who are not more than thirteen years of age. The unit has four consultant pediatric surgeons, resident doctors that pass through on rotation and house officers who come there to pursue their houseman ship. The unit has two principal nurses in charge of the unit, two senior nurses, some nursing officers and student nurses who are posted to the unit to undertake their practical training.

A prospective study was preceded with a four days piloting of the data collection tool for accessing the pain in the children this was from the $21^{\text {st }}$ of October, 2014 to the $24^{\text {th }}$ of October 2014. During the piloting period, certain factors which were earlier on omitted from the data collection tool (appendix 2) such as; indication for the surgical procedure,, the date on which the assessment was being done, and a session for comments was identified and so the data collection tool (appendix 2) was modified.

The study actually started on the $3^{\text {rd }}$ of November, 2014 and ended on the $7^{\text {th }}$ of November 2014.

The study included all patients who had undergone a surgical procedure within 24 hours of the $3^{\text {rd }}$ of November and the subjects were monitored for seventy-two hours since that is the duration for managing most post operative pain in children who have undergone surgery at the ward. The study excluded all patients who had undergone surgery 24 hours before $3^{\text {rd }}$ of November, 2014.

Data collection was in two parts. The first part, involved the administration of a questionnaire (appendix 1) to the Doctors and nurses at post on the ward during the study period.

The questionnaire was in three parts. The first part looked at the Demographics of the clinical care staff (Doctors and Nurses) the second part sought to know the choice and the reason for analgesics that are prescribed for post operative pain management and the third part sought the knowledge of the clinical staff about the assessment of pain in their patients.

The second part of the data collection involved the use of a data collection tool (appendix 2) designed to assess the pain of the patients. As a guide, FLACC pain assessment tool (appendix 3) and the NIP's pain scale (appendix 4) was used to assess the pain of patients on admission. The assessment of pain was done every six to eight hours since that was when most of the patients had their analgesics administered. As part of data collected, it was recorded when analgesics were administered or not administered.

The study was for academic purposes and was fully anonymous but very well explained to all stake holders, ethical approval was not sorted for. This was because I could not have enough time to meet the requirements of my institutions ethical approval. Also patients were not asked to sign informed consent. 
South American Journal of Clinical Research

Special Edition 2016

\section{Results}

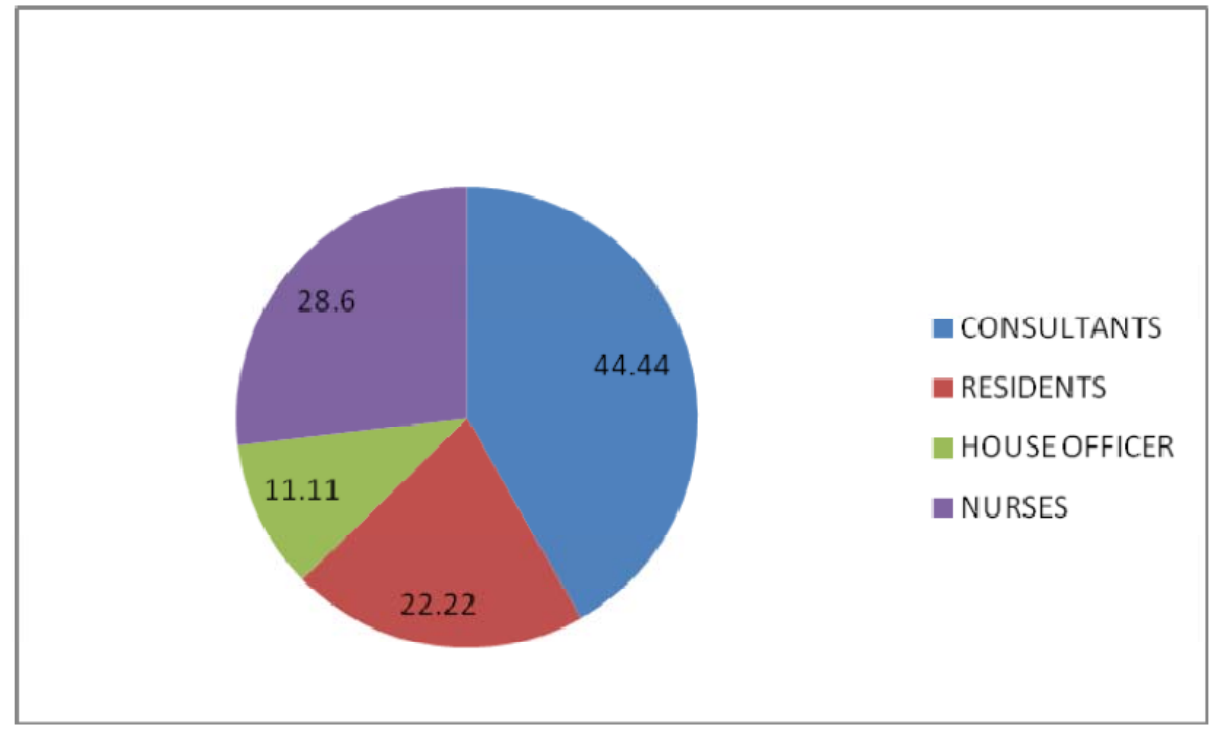

Characteristics of Health Care professionals at the Pediatric surgery ward

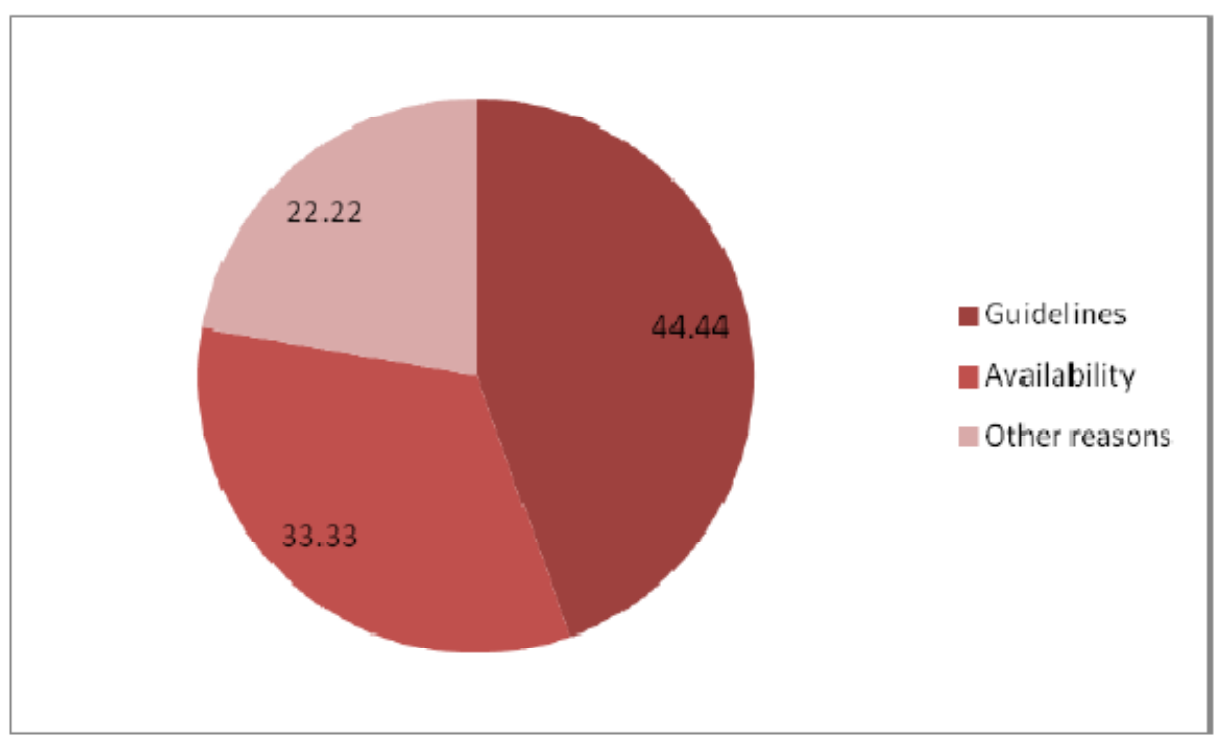

Reasons for clinicians’ first choice analgesic used post surgeries. 


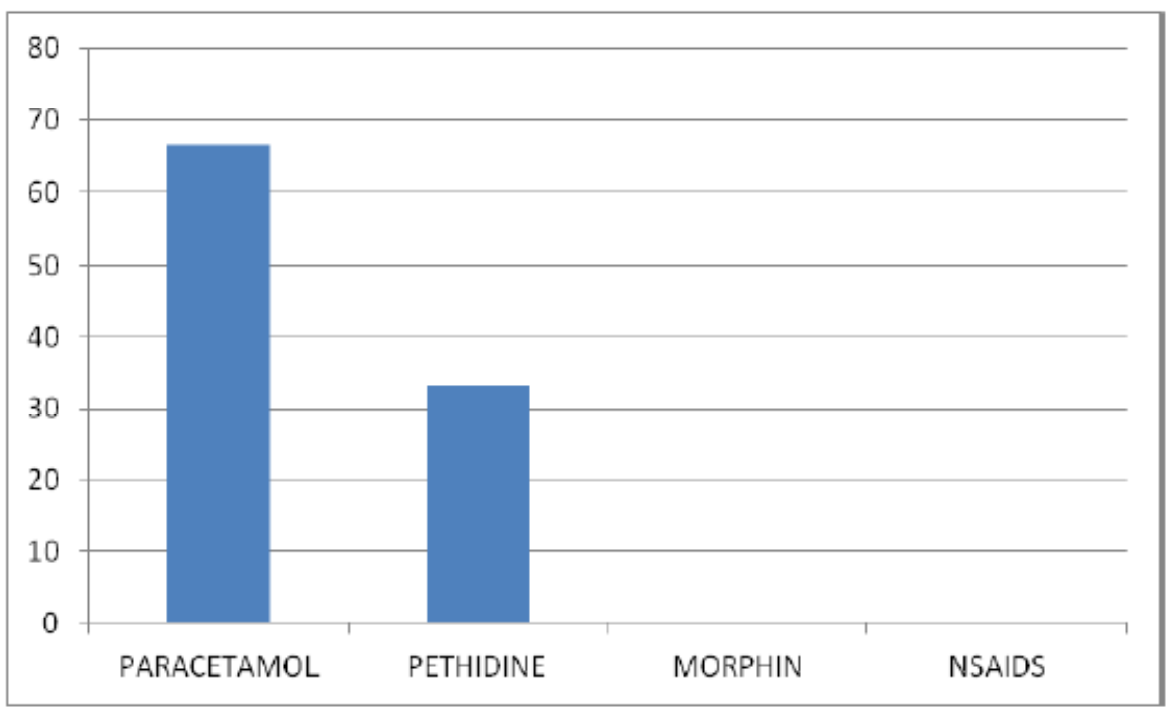

First choice analgesic used at the pediatric surgery ward of KBTH

Characteristics of patients on the pediatric surgery ward.

\begin{tabular}{|l|l|l|}
\hline Age of Patient & Number & Percentage \\
\hline Neonate & 4 & 36.4 \\
\hline $1-3$ years & 4 & 36.4 \\
\hline 4-7 years & 3 & 27.3 \\
\hline
\end{tabular}

The total number of patients on the ward during the study period was 11 and out of this, $72.7 \%$ were males and $27.3 \%$ were females

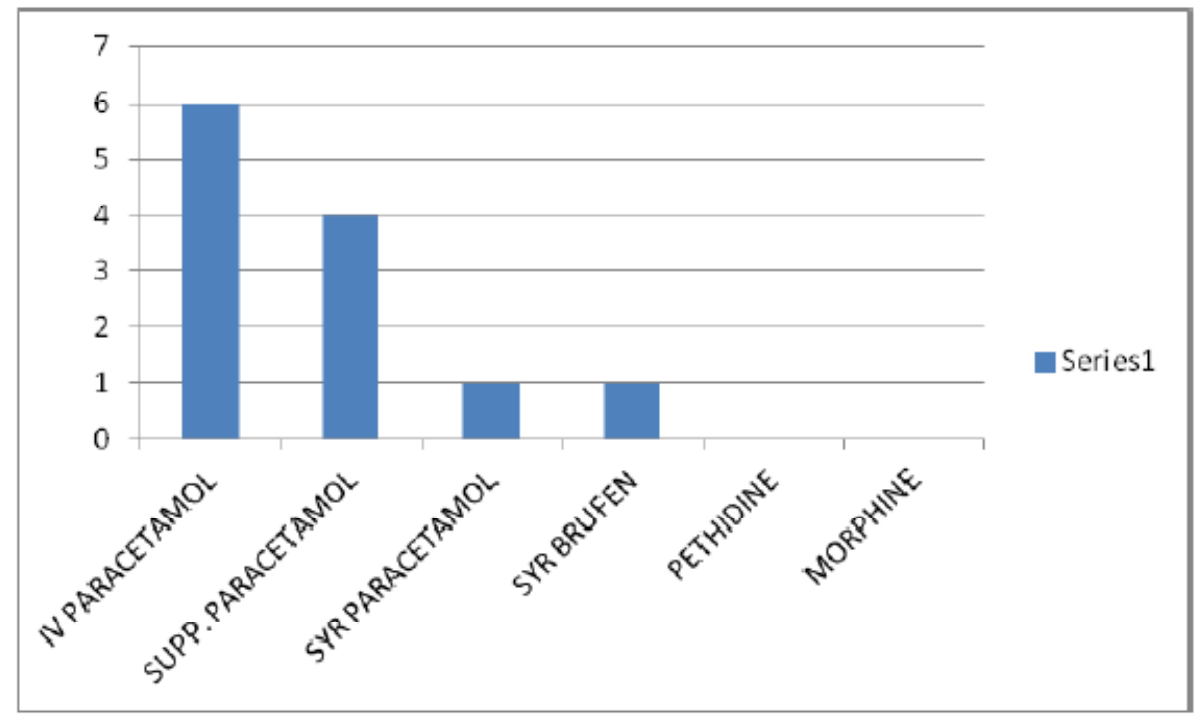

Types of Analgesic given at post op at the pediatric surgery ward. 


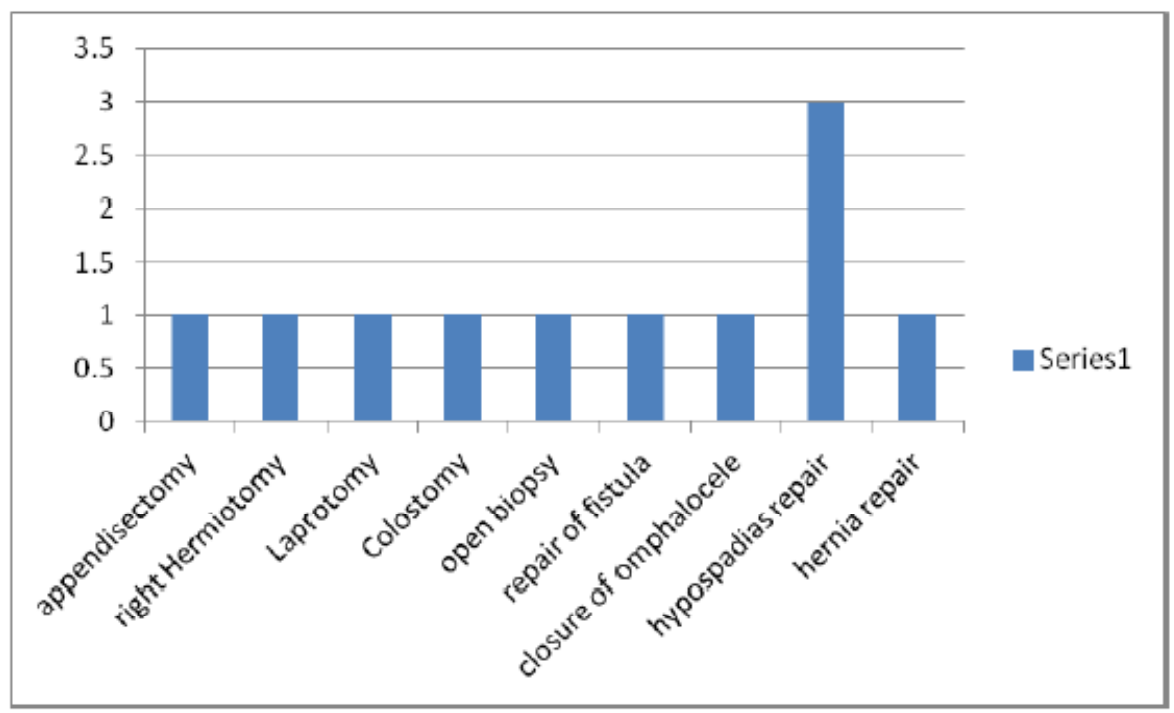

Types of surgical interventions done within the period

\section{Discussion}

There was a total of 7 clinicians and 4 nurses on the Pediatric surgery ward as of the time of the study. Marjority of the clinicians were consultants and only 2 of the nurses accepted to answer the questionnire. It was observed that most of the clinicians said their first choice post operation analgesic would be paracetamol the nurses said if they were allowed to prescribe, the would have prescribed pethidine as the first choice analgesic for post operative pain management.

The question of what informed the choice of analgesics by clinicians and nurses for the patients, $44.44 \%$ which was the highest ascribed their choice to guidelines but unfortunately there was no written down guidelines or protocol any where on the ward with information on analgesic choices for post operative pain management.

Considering the duration for post operative analgesic therapy, $77.78 \%$ of the clinicians and nurses said that they would give analgesic until patient has no pain anymore before they stop.

This study brought out the fact that no clinicians did not prescribe opiod analgesics as first choice for their patients and the reason some gave was that even when they prescribe the opiod analgesics the nurses are reluctant to give.

When it came to pain accessment of the patients it was realised there was no regular pain accessment for the patient on the ward once their analgesic was being given nobody looked out for expressions of pain in the patients. As for the use of the pain scale for the accessment of patients pain it was totally absent. Also $88.89 \%$ of the clininicians and nurses attested to the fact that they prefered accessing pain level by either talking to the patient or to the parent and only $11.11 \%$ mentioned that they would want to use the pain scale to access pain level of the patients. Also the Kokinsky and Thornberg guide to post operative pain control in children had stated that a good postoperative care in the hospital should involve a professional management of pain with the help of nurses experienced in the accessment and administion of suitable analgesics in order to reduce the discomfort of pain afterient the surgery. Also for out- patient cases, the patients parents or guardians are suppose to be given the necessary information as to how to access and manage the post operative pain in the patient when they get home. Unfortunately, this suggeston by the above guideline is not being adhered to on the pediatric surgery ward since there was no trained professional helping with the management of pain on the ward. 
The only indication used as assessment for pain was excessive crying or wailing in the children. There was an instance during the study when a patient who had undergone Right Hermiotomy was being given only suppository paracetamol for 48hours after surgery but when the patient's pain level was assessed it was at 8 . Then the data collector alerted the clinician on the ward of the need to have the patients analgesics reviewed and this was done by adding Ibuprofen to the analgesics after which the pain assessed went as low as 0 . This was not fare on the child.

Comparing this study to one conducted in 1983 by Marther and Markie, almost every child who underwent surgery had an analgesic prescribed and given to him/her within twenty four hours post op; but in the 1983 study 16\% of the patients did not have analgesic prescribed for them at all.

Most of the patients who had 'around the clock'analgesic given had a pain level as low as 0 . But most of those who kept missing their Intravenous Paracetamol due to difficulty in line assess or unavailability of the drug had higher levels of pain.

One obviouse observation was the fact that most of the clinicians and nurses did not usually consider the possible non-pharmacological means by which they could reduce pain in the patients. even though most of the stated that distracting the patient's attention could help no one ever made the attempt to use that method to control pain in the patients who appeared to be in pain even after analgesics have been given.

Looking at the surgical interventions done during the period, Hypospadias repair was the highest number of intervention done and in most of these patients pain scores were as low as 0 even on post -op day 1 with patients being given only rectal or oral paracetamol. According to the Martha and Merkie guideline it may be necessary to add Intravenous Opiod medications to the analgesic combination for such patients but it looked just the use of Acetaminophen was enough pain relief for the patients who underwent such surgeries.

\section{Conclusion}

The post operative need of the patient who undergo surgery at the pediatric surgery ward of the Korle-bu Teaching Hospital is somehow being met but much work has to be done to improve it. Patients are never well assessed for their post operative level when they get to the ward.

\section{Recommendation}

1. The should be a trained professional available at the ward at all times to help with the accessment and management of post operative pain in the patients.

2. There is a need to develop a pain management guideline for the ward where the different types of surgeries and the suggested analgesics required for pain management would be stated. As part of this written protocol, it should be mandatory to have the pain level on the ward assessed and recorded at regular intervals

3. Since research has indicated that Intravenous Acetaminophen works better and faster that the rectal and oral route administered ones, it would be very expedient for the pharmacy department of the hospital to procure some for the Pediatric pharmacy unit so it would be readily available for use.

\section{References}

[1]. Anand KJS, Carr DB (1989). The neuroanatomy, neurophysiology, and neurochemistry of pain, stress, and analgesia in newborns and children. Pediatr Clin N Am ;36: 795-822

[2]. Coll, A.M Ameen, J.R. and Mead D. (2004). Post operative pain assessment tools in day surgery literature review. Journal of advance nursing. 46(2), 124-133

[3]. D Baroudi, (2007), Post Operative Pain Management In Children. The Internet Journal of Health. (7): 2.

[4]. Fisher SM. (2000) Postoperative pain management in pediatrics. Br J Perioper Nurs. Feb; 10(2):80-4 


\section{South American Journal of Clinical Research Special Edition 2016}

[5]. Fisher SM. (2000) Postoperative pain management in pediatrics. Br J Perioper Nurs: the Journal of the National Association of Theatre Nurses 10(2): 80-84

[6]. Goldschneider et al, (2001) Pain and its management in children. In: Loeser JD, ed. Bonica’s Management of pain 3rd ed. Philadephia, PA: Lippincott Willian and Wilkings

[7]. http://www.articlesbase.com/medicine-articles/what-is-postoperative-pain-604553.html

[8]. Jacques E.(2009), CR IES Scale - Pain assessment tool, CR IES Observer Pain scale.

[9]. Jacques E.(2009) McGill Pain scale for pain assessment- McGill Pain questionnaire

[10]. Jacques E.(2009), Comfort Scale for Pain Assessment - comfort observer pain scale.

[11]. Jacques E.(2009), Numerical Pain Assessment tool for person in pain

[12]. Jacques E.(2009), Wong Baker Faces Pain scale A Pain assessment tool used by people in pain.

[13]. Kokinsty, E. and Thornberg E. (2003). Post operative pain control in children.

[14]. Lee, J.Y and Jo YY (2014) Attention to post operative pain control in children Korean J Anesthesiol, Mar; 66(3):1838.10.4097/kiae.2014.66.3.183 Epub.2014 march 28.

[15]. Marther L, Mackie J. The incidence of post operative pain in children. Pain 1983;15: 271-82

[16]. Matthews (2008) Management of post operative pain in children, U.S. Pharm 33(3): H S10-HS15 MedlineWeb of Science

[17]. Schechter NL, Allen DA, Hanson K status of pediatric pain control: a comparison of hospital analgesic usage in children and adults. Pediatrics 1986: 77:11-15

[18]. Sutters et al, (2004) A randomized clinical trial of the effectiveness of a scheduled oral analgesic dosing regimen for the management of post operative pain in children following tonsillectomy, Pain: 110;49-55

[19]. Taddio A et al, 1994 Effect of neonatal circumcision on pain responses during vaccination in boys. Lancet 345:291-2

[20]. Walco GA, Cassidy RC, Schechter NL Pain, Hurt and Harm: the ethics of pain control in infants and children. N Engl J

Med 1994;331: 541-544

[21]. Wong et al (2001) Essentials of Pediatric Nursing ed. 6, St. Louis, 1301 copyrighted Inc

\section{Acknowledgements}

All the clinicians and nurses at pediatric surgery ward $(\mathrm{kbth})$

Clin. Pharm. Obedia seaneke (head of pediatric unit pharmacy, kbth)

Clin. Pharm Frankline Acheampong (Surgical Medical Emergency Unit Pharmacy, KBTH) 